\title{
A Compact Second-order Chebyshev Bandpass Filter Using U-shaped Resonator and Defected Ground Structure
}

\author{
Nyangwarimam Obadiah ALI ${ }^{1}$, Mohammad Rijal HAMID ${ }^{2}$, Mohammad Kamal Abd RAHIM ${ }^{2}$, \\ Noor Asniza MURAD ${ }^{2}$, Sadiq THOMAS \\ ${ }^{1}$ Computer Engineering Dept., Faculty of Engineering, Nile University of Nigeria Plot 681,Cadastral Zone C-OO, \\ Research \& Institution Area, Jabi Airport Bypass, Abuja, Nigeria \\ ${ }^{2}$ Division of Communication Engineering, Advanced RF and Microwave Research Group (ARFMRG), School of \\ Electrical Eng., Faculty of Engineering, Universiti Teknologi Malaysia (UTM), Johor Bahru, Johor, 81310 Malaysia \\ ali.obadiah@nileuniversity.edu.ng, \{rijal, mkamal, asniza\}@fke.utm.my, sadiqthomas@nileuniversity.edu.ng
}

Submitted October 14, 2019 / Accepted March 16, 2020

\begin{abstract}
A compact bandpass filter using U-shaped resonators and Defected Ground Structures is proposed and designed at $5.8 \mathrm{GHz}$. The U-shaped resonators are placed around an indirectly coupled feed line while the Defected Ground Structures are positioned beneath them. The Ushaped resonator and U-shaped Defected Ground Structure are responsible for the high and low band rejection respectively. The proposed bandpass filter obeys the second order Chebyshev response which has low insertion loss of - $1.87 \mathrm{~dB}$, high rejection level and a sharp roll-off performance. The design is carried out using CST Microwave studio. The design is verified by fabricating and measuring the prototype in the laboratory. A good agreement is observed between the simulated and measured results.
\end{abstract}

\section{Keywords}

Bandpass filter, Chebyshev response, filter synthesis, high selectivity, U-shaped resonator, Defected Ground Structure (DGS)

\section{Introduction}

Communication transceiver utilizes bandpass filters to accept/reject the signals needed by devices. Research works in improving filter performances have been a challenging task to researchers. Filters are generally designed using coupled resonators of different shapes, sizes and configuration [1]. The filter configuration is done in a manner to achieve good filter performance such as low insertion loss, good selectivity and miniaturization. Various parallel coupled bandpass filters have been reported in literature, each having unique characteristics. The order of the parallel coupled filter is usually increased to improve the selectivity as seen in [2-4]. However from the reported literature, increasing the filter order also increases the overall filter size and results in increase in insertion loss. In [5-7], the parallel coupled stub is center-tapped to form a T-shaped stub and further improve the filter selectivity. Center-tapping the parallel coupled stub creates a single transmission zero. This design achieves only a single transmission zero on either side of the passband. Two transmission zeros at both high and low edges of the passband are obtained from the T-shaped stub filter shown in [6]. The parallel coupled filter is center-tapped at both sides with different unsymmetrical lengths of the stub. From the parallel coupled bandpass filters reported, the selectivity is improved by introducing stubs on the parallel coupled resonators.

A microstrip bandpass filter using stepped impedance resonator (SIR) and defected ground structure (DGS) is presented in [8], it has a good performance however the transition band on the higher band is quite high. In [9], a U-shaped DGS and two coupled U-shaped resonators are used in the design of a bandpass filter. The selectivity of the filter is quite slow. A bandpass-bandstop filter using U-shaped DGS and U-shaped cascaded resonator is presented. The filter possesses a sharp selectivity with low insertion loss. Consequently a trade-off is observed between key filter performances such as insertion loss, size and selectivity.

However, in this paper, an enhanced technique for improving the selectivity of the parallel coupled resonator is proposed. This is achieved by center offsetting and transforming the parallel coupled resonator rather than centertapping with a stub as earlier reported. This results in a U-shaped resonator formed around an indirectly coupled feedline with good rejection in the high band edge. A pair of Defected Ground Structure (DGS) is introduced beneath the U-shaped resonator to form a good rejection at the lower band edge. The presence of DGS is usually used to enhance filter behavior by suppressing out-of-band signals. 
The designed second-order bandpass filters when compared with the bandpass filter in [10-12] are found to be compact due to the method of coupling implemented (DGS).

\section{Theoretical Design of Filter}

The U-shaped resonator used in the bandpass filter design is obtained by transforming the parallel coupled resonator. The parallel coupled resonator is transformed by center-offsetting it to form a U-shaped resonator. The general equation for parallel coupled half wavelength resonator is given in (1)-(4) where $J_{j, j+1}$ is the characteristics admittance of the J-inverters, $J_{n,}{ }_{n+1}$ represents the filter inverters between the resonators, $Y_{0}$ is the characteristics admittance of the terminating lines, $F B W$ is the filter fractional bandwidth, $F_{\mathrm{U}}$ and $F_{\mathrm{L}}$ are the upper and lower band frequencies of the filter, and $g_{0}, g_{1}, \ldots, g_{n}$ represent the lowpass prototype design parameters having normalized cutoff at $\Omega_{\mathrm{c}}$ equal to 1 .

$$
\begin{gathered}
\frac{J_{01}}{Y_{0}}=\sqrt{\frac{\pi F B W}{2 g_{0} g_{1}}}, \\
\frac{J_{j, j+1}}{Y_{0}}=\frac{\pi F B W}{2} \frac{1}{\sqrt{g_{j} g_{j+1}}}, \\
\frac{J_{n, n+1}}{Y_{0}}=\sqrt{\frac{\pi}{2}} \frac{F B W}{g_{n} g_{n+1}}, \\
F B W=\frac{F_{\mathrm{U}}-F_{\mathrm{L}}}{\left(F_{\mathrm{U}}+F_{\mathrm{L}}\right) / 2} \times 100 \% .
\end{gathered}
$$

The parallel coupled resonator is shown in Fig. 1. It has a low quality factor. The parallel coupled resonator is center-offset as shown in Fig. 2 to improve the quality factor. It is observed that as the parallel coupled resonator of half-wavelength is moved away from the center a transmission zero is obtained when the offset is $10 \mathrm{~mm}$ away as shown in Fig. 1 to 3.

A sharper roll-off is obtained by doubling the centeroffset parallel coupled resonator as shown in Fig. 4. This sharper roll-off results from the strong coupling of the half wavelength resonator to the indirectly coupled feedline. The two resonators are further bent to form an L-shaped resonator. The L-shaped resonator increases the $S_{21}$ level by $8 \mathrm{~dB}$ due to the increase in coupling strength as compared

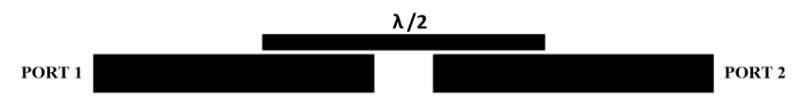

Fig. 1. Parallel coupled resonator structure (half wavelength).
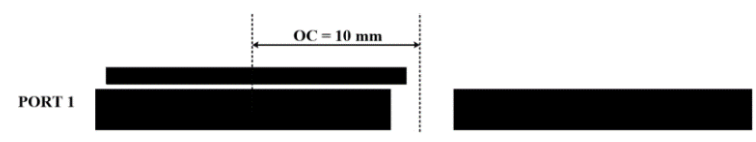

PORT 2

Fig. 2. Centre-offset parallel coupled resonator structure (half wavelength).

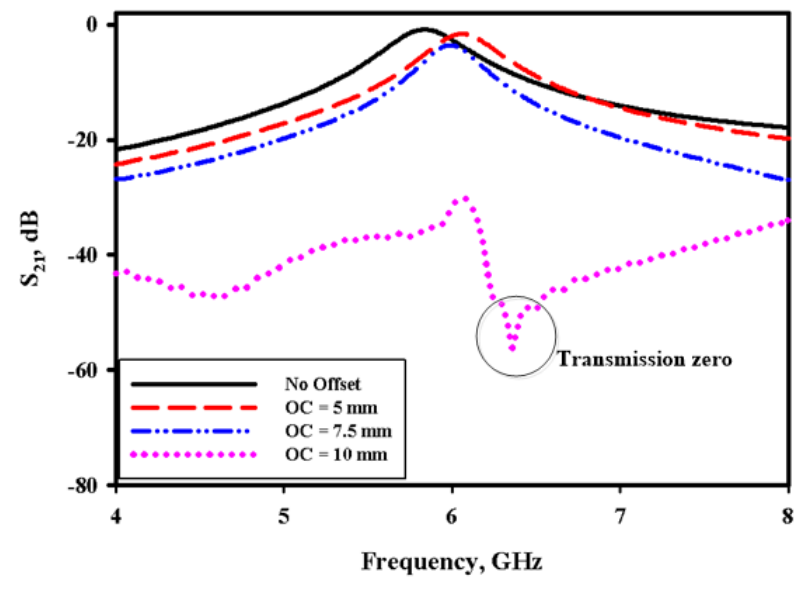

Fig. 3. Centre-offset parallel coupled resonator $S_{21}$ performance.

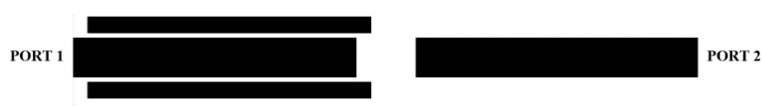

Fig. 4a. Centre-offset single and double sided parallel coupled resonator.

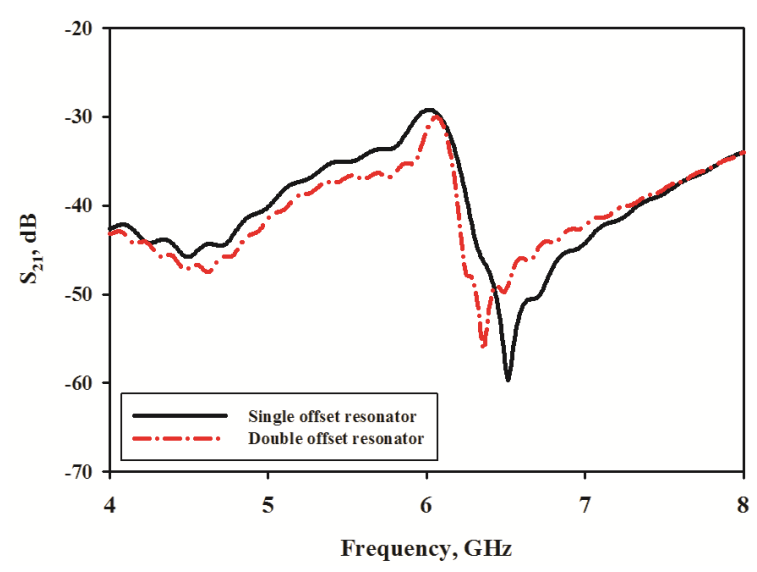

Fig. 4b. Performance comparison of center-offset single and double sided parallel coupled resonator.

with the offset. The two L-shaped resonators then combine to form a U-shaped resonator around the indirectly coupled feedline as shown in Fig. 5. However, it is noteworthy that the roll-off of the low band rejection remains poor. This issue will be addressed in Defected Ground Structure section.

\section{Filter Synthesis}

The filter synthesis technique is used to determine the external quality factor and coupling coefficient of the filter. $Q_{\mathrm{e} 1}$ and $Q_{\mathrm{en}}$ representing the external quality factor of the

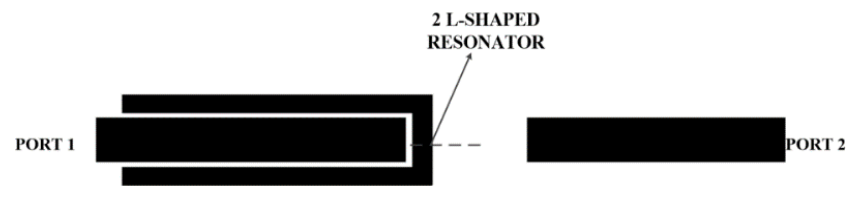

Fig. 5a. Single U-shaped resonator structure. 


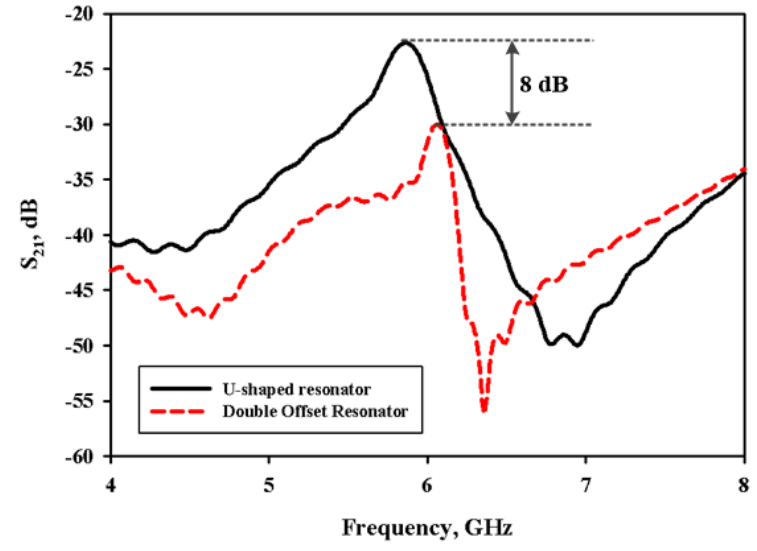

Fig. 5b. Single U-shaped resonator structure performance.

input and output of the filter. $M_{i, i+1}$ is the coupling coefficient between any two resonators. Equations (5)-(7) are used to determine $Q_{\mathrm{e} 1}, Q_{\mathrm{en}}$ and $M_{i, i+1}$.

$$
\begin{gathered}
Q_{\mathrm{e} 1}=\frac{g_{0} g_{1}}{F B W}, \\
Q_{\mathrm{e} n}=\frac{g_{n} g_{n+1}}{F B W}, \\
M_{i, i+1}=\frac{F B W}{\sqrt{g_{i} g_{i+1}}} .
\end{gathered}
$$

A second-order filter with a passband ripple requirement of $0.1 \mathrm{~dB}$ and $F B W$ of 0.03 is used in the filter design. The low-pass prototype parameter at cut-off frequency $\Omega_{\mathrm{c}}=1$, for the passband ripple is given as $g_{0}=1$, $g_{1}=0.8431, g_{2}=0.6220$ and $g_{3}=1.3554$. The calculated $Q_{\mathrm{e} 1}$ and $Q_{\mathrm{e} 2}$ is given as 18.735 while the coupling coefficient is given as 0.053 . The external quality factor is used to determine the gap, $g$ between the feedline and the first resonator. On the other hand, the coupling coefficient is used to determine the gap, $s$ between the resonators.

The arrangement in Fig. 6a is used to extract the external quality factor of the filter. The arrangement helps to determine the length between the resonator and the feedline with the Port 2 weakly coupled which implies that $Q_{\mathrm{e} 2}=\infty$. Figure $6 \mathrm{~b}$ shows the transmission coefficient magnitude response $S_{21}$ from which the external quality factor $Q_{\mathrm{e}}$ is determined from simulation. The external quality factor is given as shown in (8), where $f_{0}$ is the resonant frequency and $\Delta f$ is the $3 \mathrm{~dB}$ bandwidth as obtained from the simulation. The design curve represents the plot of $Q_{\mathrm{e}}$ against the gap $g$ as shown in Fig. 6c.

$$
\begin{gathered}
Q_{\mathrm{e}}=\frac{f_{0}}{\Delta f}, \\
M=\frac{f_{2}^{2}-f_{1}^{2}}{f_{2}^{2}+f_{1}^{2}} .
\end{gathered}
$$

The mutual coupling coefficient is determined by the arrangement shown in Fig. 7a to obtain the gap $s$. The ports 1

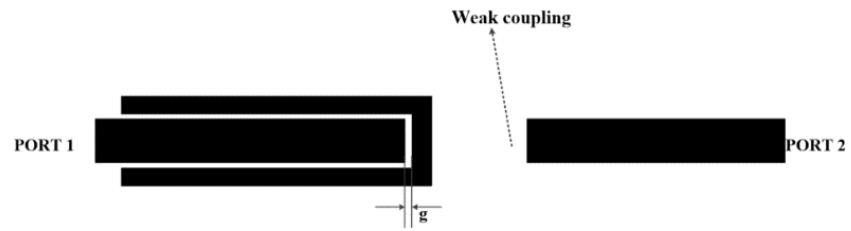

Fig. 6a. Arrangement for extraction of external quality factor.

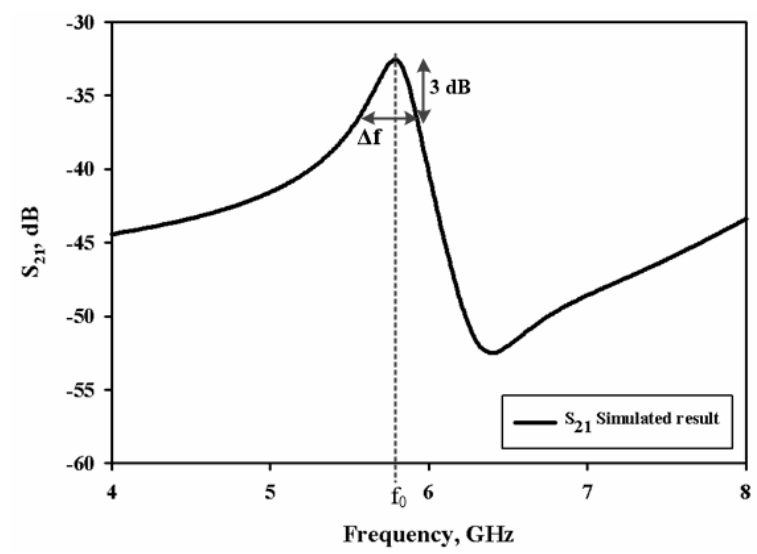

Fig. 6b. External quality factor $S_{21}$ performance.

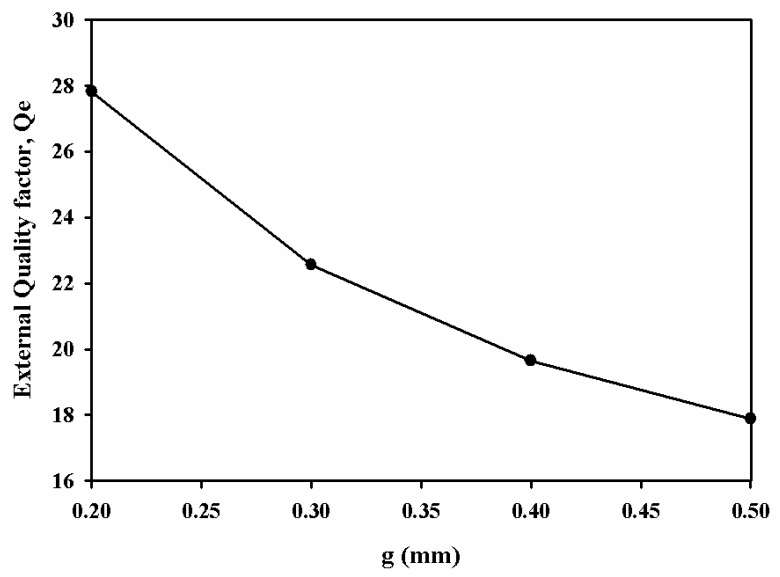

Fig. 6c. Design curve for external quality factor extraction.

and 2 are weakly coupled which means $Q_{\mathrm{e} 1}$ and $Q_{\mathrm{e} 2}$ are $\infty$. The simulation of the arrangement is used to obtain the gap between the resonators $s$. The transmission coefficient simulations $\left(S_{21}\right)$ result of the arrangement shown in Fig. 7b. The first and second peaks of $S_{21}$ are used to calculate the mutual coupling $M$ as seen in (9). The design curve in Fig. 7c shows the plot of $M$ versus length $s$.

The design curves in Fig. $6 \mathrm{c}$ and $7 \mathrm{c}$ are used to determine the filter dimensions. The filter dimensions are determined to be $g=0.4 \mathrm{~mm}$ and $\mathrm{s}=0.5 \mathrm{~mm}$. The resulting structure is shown in Fig. 8a. The performance of the filter and parallel coupled resonator line is shown in Fig. 8b. The filter is observed to have a good rejection in the high band however a poor rejection in the lower band is experienced. In order to overcome the poor rejection in the lower band a DGS is introduced to the filter structure as discussed in Defected Ground Structure section. 


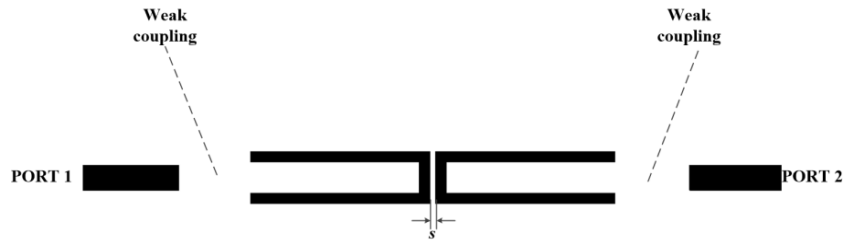

Fig. 7a. Arrangement for extraction of coupling coefficient.

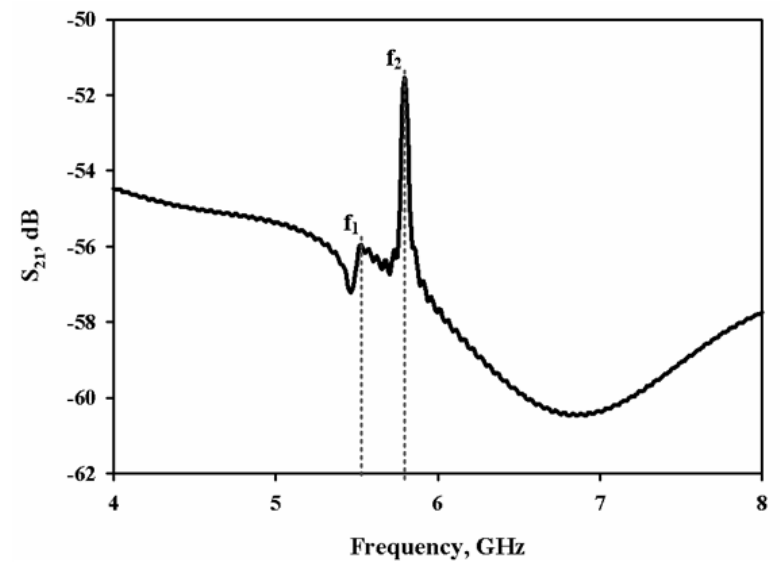

Fig. 7b. Coupling coefficient $S_{21}$ performance.

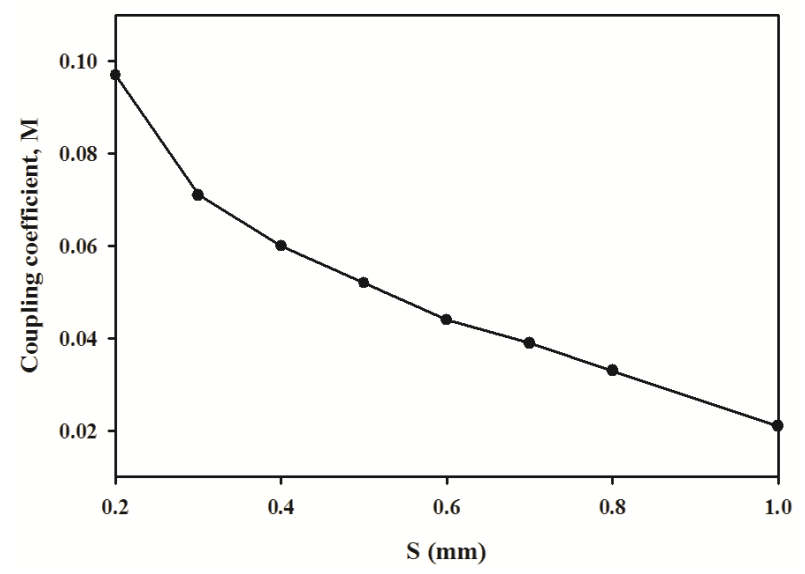

Fig. 7c. Coupling coefficient design curve.

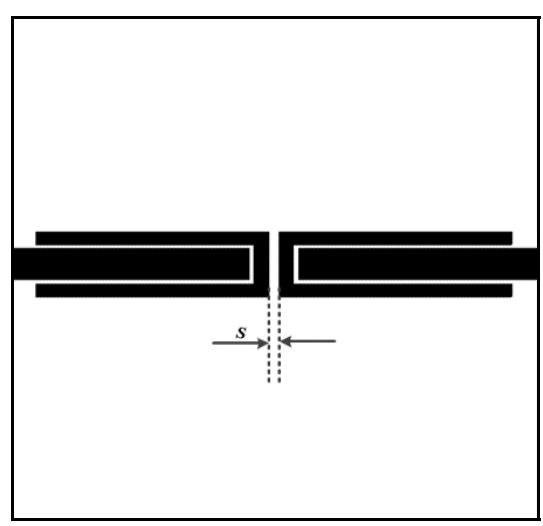

Fig. 8a. Second-order U-shaped resonator structure.

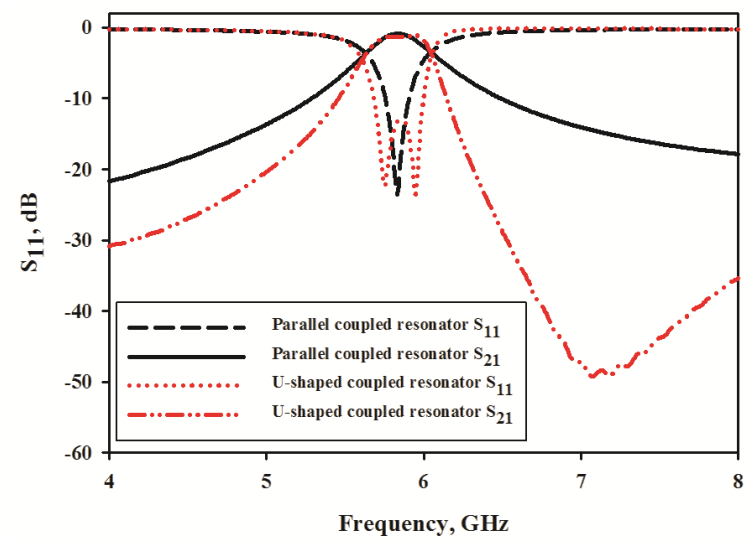

Fig. 8b. Second-order U-shaped resonator performance.

\section{Integration of Filter with Defected Ground Structure}

A pair of U-shaped DGS is used to introduce a transmission zero in the lower band of the filter. The U-shaped DGS is known for creating a single stop band [13]. The U-shaped DGS is placed directly underneath the U-shaped resonator. The structure and performance of the U-shaped DGS is shown in Fig. 9a and Fig. 9b, respectively. The parametric sweep obtained from varying the length of the DGS from $11.8 \mathrm{~mm}, 12.4 \mathrm{~mm}$ and $13.4 \mathrm{~mm}$ to yield a frequency of $5.5 \mathrm{GHz}, 5 \mathrm{GHz}$ and $4.5 \mathrm{GHz}$ respectively is shown in Fig. 9b. The DGS slot functions at half-wavelength. The DGS slot is directly proportional to the effective inductance and inversely proportional to the effective capacitance. This infers that a decrease in the DGS slot results in a corresponding decrease in the effective capacitance hence an increase in the cut-off frequency vice-versa.

Therefore a pair of U-shaped DGS is placed beneath the U-shaped resonator as shown in Fig. 10a to give a better rejection at the lower frequency band. However the matching of the filter $S_{11}$ is disturbed and noticed to be poorly matched at $-9 \mathrm{~dB}$ as shown in Fig. 10b. Hence,

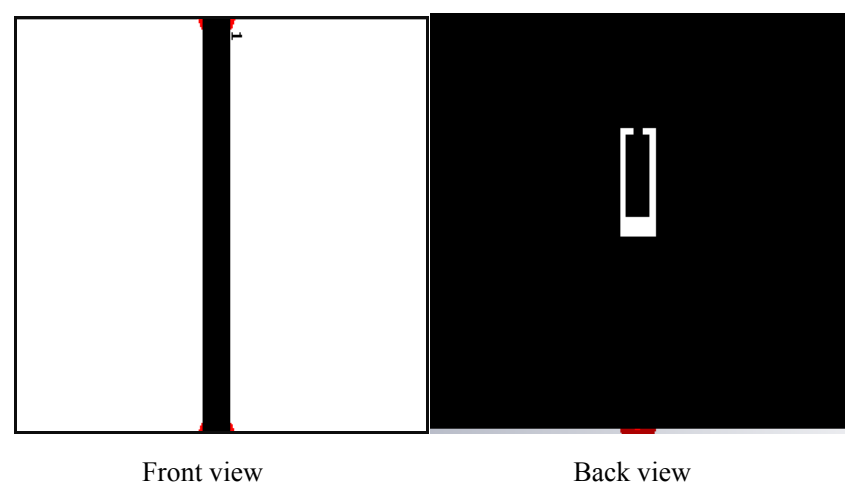

Fig. 9a. Structure for parametric sweep of U-shaped resonator. 


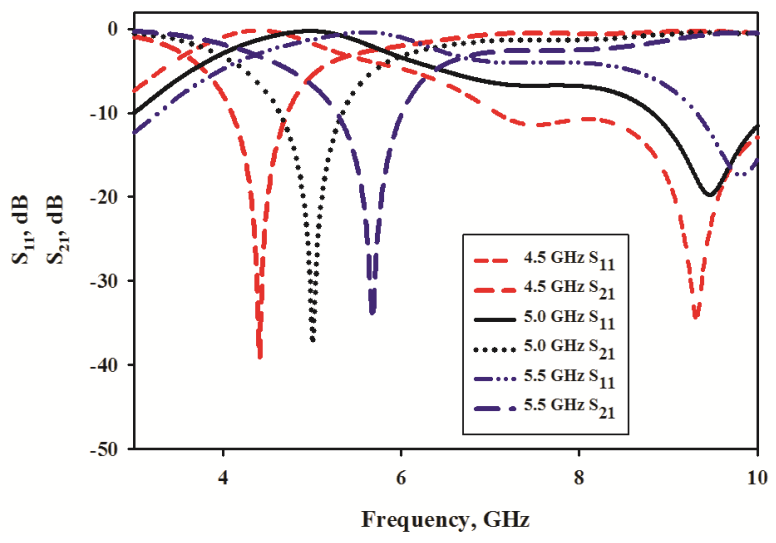

Fig. 9b. Performance for parametric sweep of U-shaped resonator.

there is the need to optimize and obtain a suitable matching. This optimization is achieved through running parametric sweep. The spacing between the two U-shaped resonators is optimized from $0.5 \mathrm{~mm}$ to $0.8 \mathrm{~mm}$ to give a good matching. Figure 10c shows the comparison of the filter performance with and without the inclusion of DGS after optimization. The filter performance with DGS gives an enhanced selectivity on both the lower and higher band of the frequency as can be seen from the $S_{21}$ result. The filter has a good rejection level of which is greater than $-49 \mathrm{~dB}$ with fast roll-off at both edges. The transmission zero on the lower passband edge and upper passband edge can be altered by modifying the length of the DGS and $\mathrm{U}$-shaped resonators respectively.

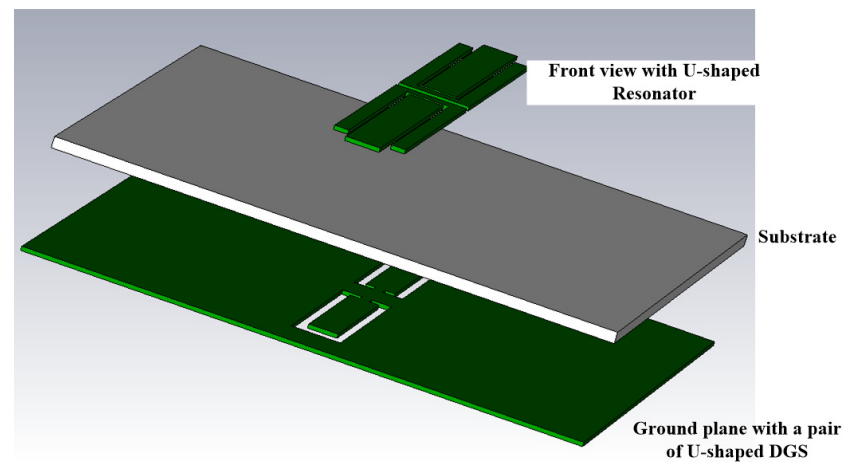

Fig. 10a. 3D filter structure with introduced DGS.

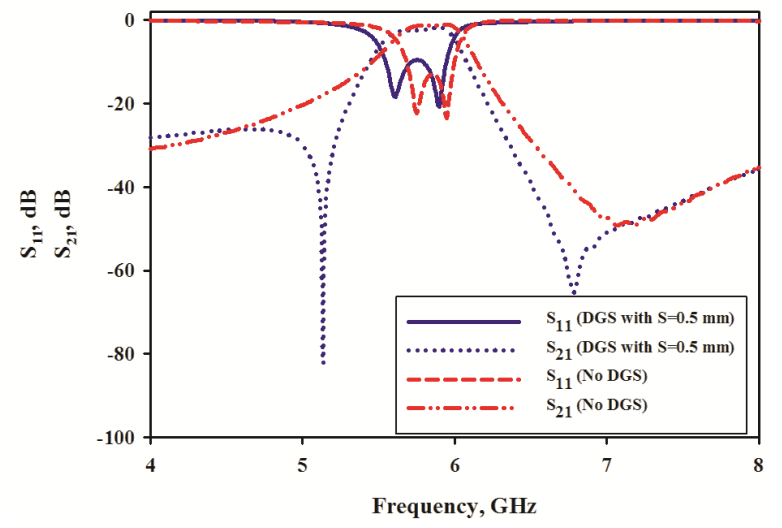

Fig. 10b. Filter performance with introduced DGS.

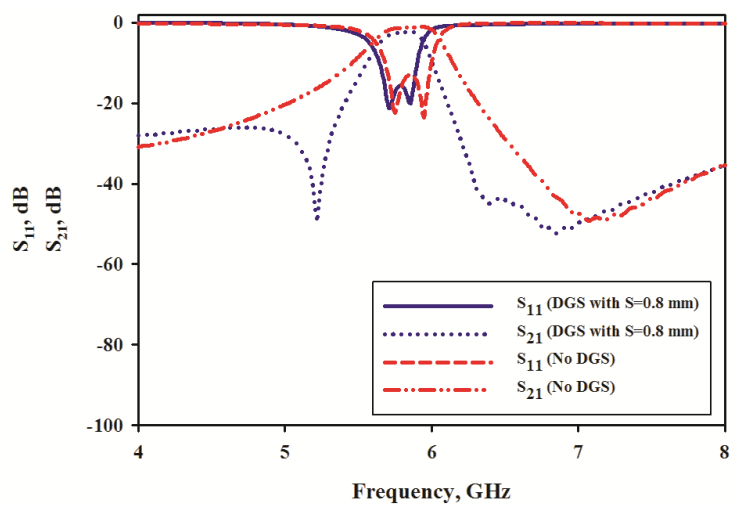

Fig. 10c. Filter performance with introduced DGS.

\section{Discussion of Results}

The bandpass filter is designed on a TLY5 Taconic substrate with a dielectric constant of 2.2 and substrate height of $0.8 \mathrm{~mm}$. The filter is designed to operate at $5.8 \mathrm{GHz}$. It is observed to have a measured low insertion loss of $-1.87 \mathrm{~dB}$, good rejection and fast roll-off at both sides of the passband. The measured passband of the filter is located between $5.65 \mathrm{GHz}$ and $5.89 \mathrm{GHz}$. The filter has two poles located at $5.66 \mathrm{GHz}$ and $5.83 \mathrm{GHz}$ as seen from the $S_{21}$ performance. The transmission zeros are located at $5.2 \mathrm{GHz}$ and $6.3 \mathrm{GHz}$ respectively. The higher band rejection results from the filter synthesis of two U-shaped resonators while the lower band rejection results from the Ushaped DGS pair. The introduction of DGS helps to improve the selectivity without increasing the filter order thereby keeping a compact filter size. The final designed filter with its parameters are shown in Fig. 11 and Tab. 1. The filter is designed and simulated using CST Microwave Studio. The simulated results are validated through fabrication of the prototype and measurements in the laboratory with the aid of a Rohde and Schwarz ZVL Network Analyzer. The fabricated prototype is shown in Fig. 12. The comparison between the simulated and measured $\mathrm{S}_{11}$ and $\mathrm{S}_{21}$ results are shown in Fig. 13, it is observed that they agree to a large extent. The little discrepancy between the simulated and measured is caused by fabrication defects and the tolerance level of the substrate used.
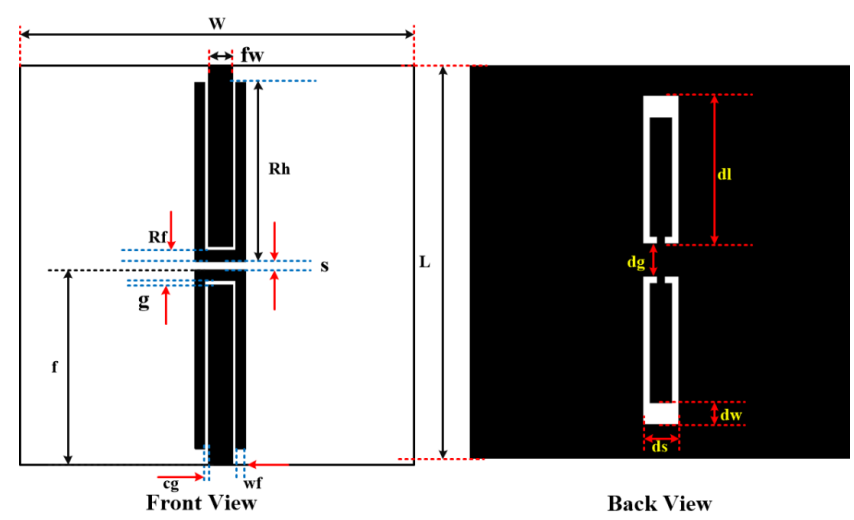

Fig. 11. Compact bandpass filter structure. 


\begin{tabular}{|l|l|l|l|l|l|l|l|}
\hline Dimensions & $\boldsymbol{W}$ & $\boldsymbol{L}$ & $\boldsymbol{g}$ & $\boldsymbol{s}$ & $\boldsymbol{R} \boldsymbol{f}$ & $\boldsymbol{R h}$ & $\boldsymbol{f}$ \\
\hline Value (mm) & 40 & 40 & 0.4 & 0.4 & 1.1 & 17.6 & 19.6 \\
\hline Dimensions & $\boldsymbol{d} \boldsymbol{s}$ & $\boldsymbol{d} \boldsymbol{w}$ & $\boldsymbol{d} \boldsymbol{l}$ & $\boldsymbol{d} \boldsymbol{g}$ & $\boldsymbol{w f}$ & $\boldsymbol{c g}$ & \\
\hline Value (mm) & 3.5 & 2 & 14.55 & 3.2 & 1 & 0.2 & \\
\hline
\end{tabular}

Tab. 1. Dimensions for filter.

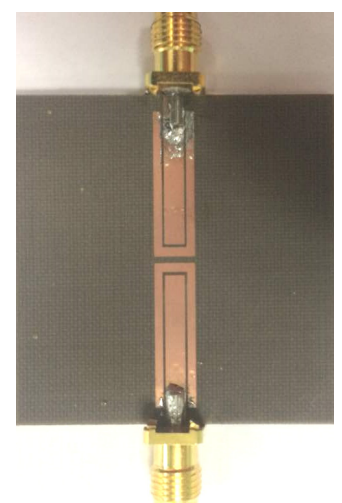

(a)

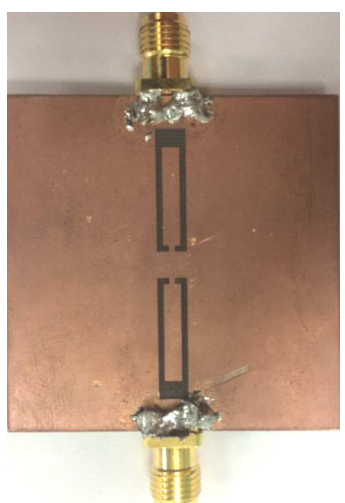

(b)
Fig. 12. Compact bandpass filter prototype.

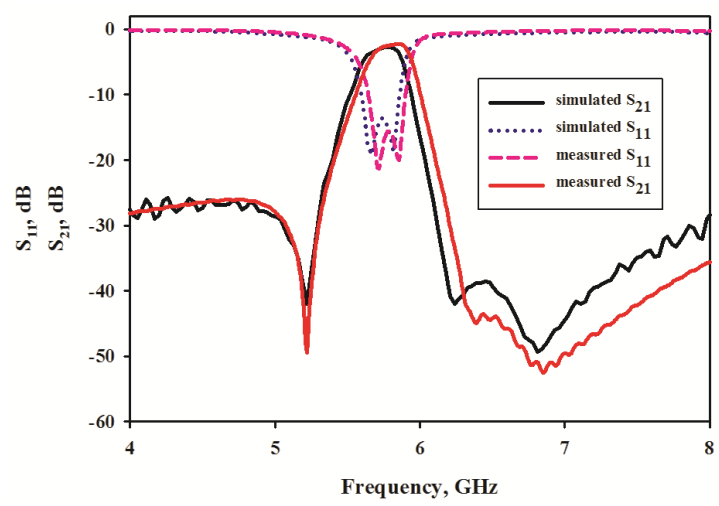

Fig. 13. Measured and simulated filter results.

\begin{tabular}{|c|c|c|c|c|}
\hline [Ref.] & $\begin{array}{c}\text { Frequency } \\
\text { (GHz) }\end{array}$ & $\begin{array}{c}\text { Guided } \\
\text { wavelength } \\
\left.\text { size( } \lambda^{2}\right)\end{array}$ & Method & Remark \\
\hline$[8]$ & 3.0 & 0.073 & SIR and DGS & $\begin{array}{c}\text { Slower rejection } \\
\text { at the higher } \\
\text { passband }\end{array}$ \\
\hline$[14]$ & 6.0 & 0.058 & $\begin{array}{c}\text { U-shaped } \\
\text { resonator and } \\
\text { DGS }\end{array}$ & $\begin{array}{c}\text { Poor rejection at } \\
\text { the lower } \\
\text { passband }\end{array}$ \\
\hline$[15]$ & 3.7 & 0.102 & $\begin{array}{c}\text { U-shaped } \\
\text { resonator and } \\
\text { DGS }\end{array}$ & $\begin{array}{c}\text { Slower rejection } \\
\text { at higher } \\
\text { passband }\end{array}$ \\
\hline$[$ This & 5.8 & 0.134 & $\begin{array}{c}\text { U-shaped } \\
\text { resonator and } \\
\text { DGS }\end{array}$ & $\begin{array}{c}\text { Sharp rejection at } \\
\text { both sides of the } \\
\text { passband }\end{array}$ \\
\hline
\end{tabular}

Tab. 2. Comparison between the proposed filter and other filters.

The comparison between the proposed filter and other filters employing similar methods of design are presented in Tab. 2. The proposed filter has the advantage of a sharp rejection on both sides of the passband as compared with others who show good rejection on a single side of the passband.

\section{Conclusion}

A compact microstrip bandpass filter is proposed in this paper. The bandpass filter operates as a second order Chebyshev response at $5.8 \mathrm{GHz}$ with a low insertion loss, good rejection and sharp roll off performance. The bandpass filter results from center offsetting a parallel coupled feedline to form a U-shaped resonator around an indirectly coupled feedline. The U-shaped resonator is responsible for the higher band rejection while the U-shaped DGS results in the lower band rejection. The bandpass filter is designed from a combination of U-shaped resonators and U-shaped DGS. The designed filter is suitable for WLAN applications.

\section{Acknowledgments}

The authors would like to thank Universiti Teknologi Malaysia (UTM) for supporting this work under the grant reference number Q.J130000.2623.14J18 and Q.J130000.3551.06G94. The authors also appreciate the Nile University of Nigeria for contributing to the viability of this research.

\section{References}

[1] HONG, J.-S. G., LANCASTER, M. J. Microstrip Filters for RF/Microwave Applications. John Wiley \& Sons, 2004. DOI: $10.1002 / 0471221619$

[2] MAKIMOTO, M., YAMASHITA, S. Bandpass filters using parallel coupled stripline stepped impedance resonators. IEEE Transactions on Microwave Theory and Techniques, 1980, vol. 28, no. 12, p. 1413-1417. DOI: 10.1109/TMTT.1980.1130258

[3] KUO, J.-T., CHEN, S.-P., JIANG, M. Parallel-coupled microstrip filters with over-coupled end stages for suppression of spurious responses. IEEE Microwave and Wireless Components Letters, 2003, vol. 13, no. 10, p. 440-442. DOI: 10.1109/LMWC.2003.818531

[4] CHEONG, P., FOK, S.-W., TAM, K.-W. Miniaturized parallel coupled-line bandpass filter with spurious-response suppression. IEEE Transactions on Microwave Theory and Techniques, 2005, vol. 53, no. 5, p. 1810-1816. DOI: 10.1109/TMTT.2005.847075

[5] LEE, J.-R., CHO, J.-H., YUN, S.-W. New compact bandpass filter using microstrip/spl lambda//4 resonators with open stub inverter. IEEE Microwave and Guided Wave Letters, 2000, vol. 10, no. 12, p. 526-527. DOI: $10.1109 / 75.895091$

[6] ZHU, L., MENZEL, W. Compact microstrip bandpass filter with two transmission zeros using a stub-tapped half-wavelength line resonator. IEEE Microwave and Wireless Components Letters, 2003, vol. 13, no. 1, p. 16-18. DOI: 10.1109/LMWC.2002.807705

[7] OBADIAH, A. N., HAMID, M. R., RAHIM, M. K. A., et al. A compact bandpass filter using a T-shaped loaded open-ended stub resonator. Indonesian Journal of Electrical Engineering and Computer Science, 2018, vol. 10, no. 3, p. 867-874. DOI: 10.11591/ijeecs.v10.i3.pp867-874

[8] LI, Z., W. SHI, W., YUAN, Y. A novel compacted microstrip bandpass filter using stepped impedance resonator (SIR) and 
defected ground structure (DGS). In 2014 15th International Conference on Electronic Packaging Technology. Chengdu (China), 2014, p. 1338-1340. DOI: 10.1109/ICEPT.2014.6922895

[9] BOUTEJDAR, A., BATMANOV, A., AWIDA, M. H., et al Design of a new bandpass filter with sharp transition band using multilayer-technique and $\mathrm{U}$-defected ground structure. IET Microwaves, Antennas \& Propagation, 2010, vol. 4, no. 9, p. 1415-1420. DOI: 10.1049/iet-map.2009.0357

[10] NWAJANA, A. O., DAINKEH, A., YEO, K. S. K. Substrate integrated waveguide (SIW) bandpass filter with novel microstripCPW-SIW input coupling. Journal of Microwaves, Optoelectronics and Electromagnetic Applications, 2017, vol. 16, no. 2, p. 393-402. DOI: 10.1590/2179-10742017v16i2793

[11] MOHOTTIGE, N., GLUBOKOV, O., JANKOVIC, U., et al. Ultra compact inline E-plane waveguide bandpass filters using cross coupling. IEEE Transactions on Microwave Theory and Techniques, 2016, vol. 64, no. 8, p. 2561-2571. DOI: 10.1109/TMTT.2016.2578329

[12] RABAHALLAH, D., CHALLAL, M., TALAHARIS, N. Tri-band microstrip bandpass filters for GSM and WiMAX applications. In 2015 4th International Conference on Electrical Engineering (ICEE). Boumerdes (Algeria), 2015, p. 1-4. DOI: 10.1109/INTEE.2015.7416830

[13] KHANDELWAL, M. K., KANAUJIA, B. K., KUMAR, S. Defected ground structure: Fundamentals, analysis, and applications in modern wireless trends. International Journal of Antennas and Propagation, 2017, p. 1-22. DOI: $10.1155 / 2017 / 2018527$

[14] BOUTEJDAR, A., ELHANI, S., BENNANI, S. D. Design of a novel slotted bandpass-bandstop filters using U-resonator and suspended multilayer-technique for L/X-band and Wlan/WiMax applications. In International Conference on Electrical and Information Technologies (ICEIT). Rabat (Morocco), 2017, p. 1-7. DOI: 10.1109/EITech.2017.8255272

[15] BOUTEJDAR, A., BATMANOV, A., AWIDA, M. H., et al. Design of a new bandpass filter with sharp transition band using multilayer-technique and U-defected ground structure. IET Microwaves, Antennas \& Propagation, 2010, vol. 4, no. 9, p. 1415-1420. DOI: 10.1049/iet-map.2009.0357

\section{About the Authors...}

Nyangwarimam Obadiah ALI received his Bachelor of Engineering (B.Eng.) in Electrical and Computer Engineering from the Federal University of Technology Minna, Niger state, Nigeria in 2010 and a Master of Engineering (M.Eng.) in Electrical-Electronics and Telecommunication Engineering from the University of Technology, Malaysia in 2014. He received a Ph.D. in Electrical Engineering from the University of Technology, Malaysia in 2018. He was with the Advanced RF and Microwave Research Group, Division of Communication Engineering, School of Electrical Engineering, Faculty of Engineering, Universiti Teknologi Malaysia. He is now a Lecturer with the Dept. of Computer Engineering, Faculty of Engineering, Nile University of Nigeria, Abuja, Nigeria. His research interest includes radio frequency devices, reconfigurable antennas, filters and filter-antennas.

Mohamad Rijal HAMID received the M.Sc. degrees in
Communication Engineering from the Universiti Teknologi Malaysia, Johor Bahru, Malaysia, in 2001 and the Ph.D. Degree at the University of Birmingham, Birmingham, U.K. in 2011. He has been with Universiti Teknologi Malaysia (UTM) at the Communications Engineering Division, School of Electrical Engineering, Faculty of Engineering, UTM, since 1999. Currently his position is a Senior Lecturer. His major research interest is reconfigurable antenna design for multimode wireless applications. $\mathrm{He}$ was awarded a scholarship from the Universiti Teknologi Malaysia to further study in the U.K.

Mohamad Kamal A. RAHIM was born in Alor Setar, Kedah, Malaysia in 1964. He obtained his B.Eng. in Electrical and Electronic from the University of Strathclyde, U.K. in 1987 and M.Eng. from the University of New South Wales, Australia in 1992. He received his Ph.D. in the field of wideband active antenna. He is a Professor at Communications Engineering Division, School of Electrical Engineering, Faculty of Engineering at Universiti Teknologi Malaysia. Professor Mohamad Kamal is a senior member of IEEE since 2007. His research interest includes antennas, metamaterials, body-area communications, and reconfigurable antennas vertically.

Noor Asniza MURAD obtained her first degree in 2001 from Universiti Teknologi Malaysia (UTM), Malaysia, with Honors, majoring in Telecommunication Engineering. Shortly after graduated, she joined UTM as a tutor attached to the Department of Radio Communication Engineering (RaCED), Faculty of Electrical Engineering (FKE), UTM. She received her M.Eng. in 2003 from the same university and later has been appointed as a Lecturer in April 2003. She joined Emerging Device Technology Group, University of Birmingham, UK and obtained her Ph.D. in 2011 for research on micro-machined millimeter wave circuits. Her research interests include antenna design for RF and microwave communication systems, millimeter wave circuits design, and antenna beamforming. Currently, Noor Asniza Murad is a member of IEEE (MIEEE), Member of Antenna and Propagation (AP/MTT/EMC) Malaysia Chapter, and a Senior Lecturer at Communications Engineering Division, School of Electrical Engineering, Faculty of Engineering at Universiti Teknologi Malaysia (UTM).

Sadiq THOMAS was born in 1980, and is an Associate Professor of Computer Engineering and the team leader for the C\&E research group at Nile University of Nigeria, Abuja. He has several years' experience in academics from time spent completing his B.Eng. in Computer Engineering 2005 and Numerical Modelling of EMATs for his Ph.D. thesis. He had his post-doctoral research and lecturing posts at various universities in the UK and Nigeria. He completed his Ph.D. in 2009 and his research was based on comprehensive finite element modelling of electromagnetic acoustic transducer sensors used for nondestructive testing of conductive materials particularly useful for pipeline maintenance. He is also involved in several research areas, which includes communication and sensor technology. 